\title{
Triangulação de métodos: Um caminho para a Avaliação de projetos de educação permanente em Saúde
}

\author{
Gilmara Apolinário Reis', Marta Azevedo dos Santos ${ }^{1}$, Caroline \\ Roberta Freitas Pires ${ }^{1}$, Neilton Araújo de Oliveira ${ }^{1}$, Eduardo José \\ Cezari' e Danielle Keilla Alencar Cruz ${ }^{2}$
}

\begin{abstract}
1 Universidade Federal do Tocantins, Brasil | gil_apr@uft.edu.br; marta@uft.edu.br; carolinerfpires@uft.edu.br; neilton@uft.edu.br; eduardo@uft.edu.br | https://orcid.org/00000001-8113-9300; https://orcid.org/0000-0002-3219-8555; https://orcid.org/0000-00021427-7276; https://orcid.org/0000-0002-8976-7237; https://orcid.org/0000-0001-7909-1504

2 Ministério da Saúde, Brasil | daniellealencruz@gmail.com | https://orcid.org/0000-0001. 5635-5378
\end{abstract}

\begin{abstract}
Resumo: No Brasil, o programa Academia da saúde representa uma das principais estratégias para o enfrentamento ao avanço das DCNT, por isso, visando seu fortalecimento, o Ministério da Saúde (MS) desenvolveu em parceria com a Universidade Federal do Tocantins (UFT), um caderno técnico e uma formação presencial, a partir de um processo pedagógico participativo baseado em metodologias ativas de ensino-aprendizagem. Com isso posto, a pergunta colocada foi se a triangulação de métodos, possibilita uma avaliação de projetos de educação permanente e um revisitar das várias possibilidades metodológicas na pesquisa qualitativa. $O$ presente artigo objetiva analisar e descrever como a estratégia de triângulação de métodos foi empregada para o desvelar de ambos os processos formativos. Trata-se de uma pesquisa educacional de natureza qualitativa, do tipo pesquisa-ação, com enfoque crítico-participativo, delineamento exploratório-descritivo e recorte transversal. A coleta de dados ocorreu no período de maio a dezembro de 2019, sendo utilizada a estratégia de avaliação por triangulação de métodos qualitativos (observaçãoparticipante, análise documental e entrevista com informante-chave). Para a análise dos dados foram utilizadas as técnicas de análise de conteúdo proposta por Bardin e análise documental. A entrevista com informante-chave permitiu resgatar o processo de construção do caderno técnico, já os achados observacionais e documentais permitiram refazer o trajeto percorrido pelos educadores para a construção e aplicação da formação presencial, desde a composição da equipe interdisciplinar e implicada no quadrilátero do SUS, até as técnicas e recursos pedagógicos utilizados na formação presencial aplicada, que se mostraram alinhados à pedagogia freiriana, na qual se embasa a Política Nacional de Educação Permanente em Saúde do SUS. A estratégia de triangulação de métodos qualitativos permitiu elucidar de forma aprofundada e assertiva as etapas percorridas e as interfaces pedagógicas utilizadas, tanto na construção do caderno, como da formação presencial, a partir de perspectivas integradas, garantindo maior representação da realidade na pesquisa realizada.
\end{abstract}

Palavras-chave: Pesquisa Educacional Qualitativa; Educação Permanente em Saúde; Programa Academia da Saúde.

Triangulation of Methods: A Path for the Evaluation of Permanent Education Projects in Health

Abstract: In Brazil, the Academia da Saúde program represents one of the main strategies for coping with the advancement of NCDs, therefore, aiming at strengthening it, the Ministry of Health (MS) developed in partnership with the Federal University of Tocantins (UFT), a technical notebook and classroom training, based on a participatory pedagogical process based on active teachinglearning methodologies. With that said, the question posed was whether the triangulation of methods enables an evaluation of permanent education projects and a revisit of the various methodologica possibilities in qualitative research. The present article aims to analyze and describe how the method triangulation strategy was used to unveil both formative processes. This is an educational research of a qualitative nature, of the research-action type, with a critical-participatory focus, exploratorydescriptive design and transversal cut. Data collection took place from May to December 2019, using the triangulation assessment strategy of qualitative methods (participant observation, document analysis and interview with key informants). For data analysis, the techniques of content analysis proposed by Bardin and document analysis were used. The interview with a key informant allowed to rescue the process of construction of the technical notebook, whereas the observational and documentary findings allowed to redo the path traveled by the educators for the construction and application of the face-to-face training, from the composition of the interdisciplinary team and involved in the quadrilateral from SUS, even the pedagogical techniques and resources used in applied face-to-face training, which proved to be in line with Freire's pedagogy, on which SUS's National Policy for Permanent Education in Health is based. The triangulation strategy of qualitative methods allowed to elucidate in a profound and assertive way the steps covered and the pedagogical interfaces used, both in the construction of the notebook, as well as in-person training, from integrated perspectives, ensuring a greater representation of reality in the research carried out.

Keywords: Qualitative Educational Research; Permanent Health Education; Health Academy Program. 


\section{Introdução}

A criação do Sistema Único de Saúde (SUS) em 1988, seguida pela implantação do "Programa Saúde da Família" e a expansão da Atenção Primária na década de 90, incorporaram a promoção de saúde ao modelo assistencial brasileiro. Nos anos 2000, o Ministério da Saúde lançou diversas iniciativas voltadas à produção de hábitos de vida saudáveis, culminando na publicação da Política Nacional de Promoção da Saúde (PNPS) em 2006. Além disso, programas como o "Bolsa Família" e o "Saúde na Escola", mobilizaram diversos setores da sociedade para o enfrentamento aos Determinantes Sociais da Saúde (Buss e Carvalho, 2009).

Tal conjuntura impulsionou a implantação do Programa Academia da Saúde em 2011, por meio da Portaria GM/MS no 719. O programa representa uma das principais estratégias do Ministério da Saúde para o enfrentamento ao avanço das DCNT no Brasil, partindo do fortalecimento da Política Nacional de Promoção da Saúde (PNPS-SUS) no contexto da Atenção Primária à Saúde (APS-SUS).

O crescimento exponencial do Programa Academia da Saúde nos últimos anos tem demandado um aporte técnico por parte dos gestores no nível local, dada a diversidade de ações e a contemporaneidade dos seus objetivos frente à situação de saúde do país, exigindo saberes e práticas de saúde pouco operáveis na abordagem biomédica de cuidado que ainda vigora no SUS.

Visando o fortalecimento do Programa Academia da Saúde, o Ministério da Saúde vem desenvolvendo estratégias de educação permanente junto aos gestores e profissionais de saúde dos estados e municípios desde 2013, passando a contar com a colaboração da Universidade Federal do Tocantins a partir de 2016. Inicialmente na construção do caderno técnico de apoio à implantação e implementação do programa Academia da Saúde ${ }^{1}$, e como desdobramento deste, no desenvolvimento da formação presencial baseada nos conteúdos programáticos do caderno técnico, voltada à construção de capacidades técnicas relacionadas à gestão do programa, executada por meio da carta acordo firmada entre MS/UFT (2019/2020)2.

Tanto a construção do caderno técnico, quanto a elaboração da formação presencial, foram realizadas de forma coletiva pela equipe de pesquisadoras da UFT em parceria com a equipe técnica do Ministério da Saúde. A primeira iniciativa passou por um processo de validação em cinco estados brasileiros ${ }^{3}$, representando quatro regiões do país, escolhidos pela expertise desenvolvida no programa, com profissionais de saúde e gestores locais. Já a segunda, foi aplicada nas oito microrregiões de saúde o estado do Tocantins, contemplando um conjunto de oficinas interligadas dialeticamente e distribuídas em dois dias de atividades, utilizando metodologias ativas de ensino-aprendizagem.

Partindo deste contexto, surgiu a necessidade de avaliar o processo de elaboração do caderno técnico e da formação presencial desenvolvidos para a capacitação de profissionais do programa Academia da Saúde, com o objetivo de descrever, identificar e analisar as abordagens pedagógicas utilizadas, à luz dos pressupostos da educação permanente em saúde. Avaliação está com enfoque na triangulação de métodos.

1 O Caderno Técnico de Apoio à Implantação e Implementação do programa Academia da Saúde está disponível em: http://189.28.128.100/dab/docs/portaldab/publicacoes/implatacao_academia_saude.pdf 2 Mais informações sobre o projeto e a equipe de execução, conforme carta acordo firmada entre MS/UFT, estão disponíveis no site: https://sites.uft.edu.br/progacademiadasaude/

3 O processo de validação do Caderno Técnico do Programa Academia da Saúde ocorreu nos estados do Ceará, Pernambuco, Minas Gerais, Paraná e Espirito Santo. 
Vale ressaltar que o referencial teórico-metodológico adotado para a análise, por estar situado no âmbito da Política Nacional de Educação Permanente em Saúde, foi ancorado nos pressupostos da pedagogia freiriana, que entende o processo pedagógico como essencialmente histórico, mediado pelas relações do ser humano com o mundo e fundamentado na dialogicidade e horizontalidade entre educador-educando, autonomia e a pedagogia da experiência vivida como lugar de aprendizagem.

\section{Metodologia}

O presente estudo configurou-se como uma pesquisa educacional de natureza qualitativa, do tipo pesquisa-ação, com enfoque crítico-participativo, delineamento exploratóriodescritivo e recorte transversal, utilizando três metodologias de investigação qualitativa: observação-participante, análise documental e entrevista com informantechave, analisadas à luz das técnicas de análise de conteúdo (Bardin, 2011) e análise documental.

Este desenho foi escolhido com base nas características da pesquisa-ação, a qual possibilita a interação ativa e participativa das pesquisadoras com a equipe do projeto. Sendo assim, a coleta de dados ocorreu no período de maio a dezembro de 2019 na Universidade Federal do Tocantins (UFT) e nas cidades-sede das oito microrregiões de saúde do estado do Tocantins, durante o processo de elaboração das oficinas e a aplicação da formação presencial desenvolvida no âmbito do projeto de "construção de capacidades técnicas relacionadas à gestão do programa Academia da Saúde".

A estratégia de avaliação por triangulação de métodos é descrita por Minayo, Assis e Souza (2005), na qual são combinados vários métodos científicos visando aprofundar o conhecimento e a representação da realidade estudada. Sendo assim, a escolha das técnicas de coleta de dados foi guiada pelo objetivo principal do trabalho, que buscou analisar os processos de elaboração tanto do caderno técnico do programa Academia da Saúde, como da formação presencial desenvolvida no estado do Tocantins, à luz dos pressupostos da educação permanente em saúde e da pedagogia freiriana, com a triangulação de métodos qualitativos de investigação.

Dessa forma, para analisar o processo pedagógico de educação permanente em saúde desenvolvido no âmbito do programa Academia da Saúde, buscou-se remontar o percurso pedagógico empreendido pela equipe responsável pela elaboração do caderno, das oficinas e das metodologias, a partir de entrevistas, registros documentais e da observação-participante realizada pelas pesquisadoras durante a execução do projeto.

Para tanto, foram utilizadas as seguintes estratégias metodológicas para coleta e compreensão dos dados: 1) Entrevista guiada por um roteiro semiestruturado de perguntas abertas com a responsável pelo Programa Academia da Saúde no Ministério da Saúde à época da construção do caderno técnico e da celebração da carta acordo firmada entre MS/UFT (informante-chave); 2) Observação-participante realizada pela pesquisadora, tendo colaborado na elaboração do processo pedagógico e atuado na aplicação da formação presencial desenvolvida; 3) Análise documental das atas de reuniões do projeto, dos relatórios de campo dos tutores e do instrutivo das oficinas.

A análise da entrevista com informante-chave foi realizada por meio da técnica de Análise de Conteúdo categorial temática, proposta por Bardin. Este método científico tem como foco principal analisar as mensagens de comunicações, a partir do que foi dito nas entrevistas e observado pela pesquisadora, buscando classificar o material transcrito em temas e categorias, com a finalidade de evidenciar indicadores que permitam realizar inferências sobre a realidade (Bardin, 2011). 
Bisol (2012), ressalta que a entrevista com informante-chave, é comumente utilizada em pesquisas de campo com delimitações bem definidas em termos de instituições, pessoas ou atividades de interesse da pesquisa. Nesse contexto, os indivíduos entrevistados como informantes-chave são pessoas bem informadas e com amplo contato e envolvimento ativo com o objeto da pesquisa, dessa forma é possível "obter informações profundas, insights e explicações úteis" ao desenvolvimento do trabalho a partir da qualidade, profundidade e detalhamento da informação obtida.

Já para a análise das atas de reuniões do projeto, dos relatórios de campo dos tutores e do instrutivo das oficinas, foi utilizada a técnica de análise documental. Sobre a análise documental, Souza, Kantorski e Luis (2011), destacam que a análise documental enquanto técnica de análise de dados, empreende uma série de procedimentos de transformação do material, visando facilitar a compreensão e o uso das informações contidas nos documentos analisados. Desta forma, esta técnica concentra-se no tratamento do material, de forma a tornar a informações mais acessíveis, condensadas e contextualizadas socialmente, correlacionando-as com outras fontes de dados.

Sobre a observação-participante utilizada pelas pesquisadoras durante o desenvolvimento do processo pedagógico em questão, incluindo a participação em reuniões com a equipe técnica e pedagógica do projeto, colaboração no processo de construção das oficinas e atuação como tutora na aplicação da formação presencial nos territórios de saúde do Tocantins, Lüdke e André (1986), evidenciam o lugar privilegiado da observaçãoparticipante no âmbito das pesquisas qualitativas educacionais, sendo considerada como o principal método de investigação, na medida em que "a experiência direta é sem dúvida o melhor teste de verificação da ocorrência de determinado fenômeno", além de ser comumente associada à outras técnicas como a análise documental e entrevistas.

Ressalta-se ainda que esta pesquisa contempla a Resolução 466 de 2012 do Conselho Nacional de Saúde (CNS), quanto à submissão ao comitê de ética em pesquisa e à assinatura do Termo de Consentimento Livre e Esclarecido (TCLE), tendo sido aprovada pelo Comitê de Ética em Pesquisa da Fundação Universidade Federal do Tocantins, pelo parecer consubstanciado $n=3.454 .186 / 2019$. Somente após esta etapa foram iniciadas a coleta dos dados e a pesquisa propriamente dita.

\section{Resultados e Discussão}

Considerando o posicionamento epistemológico adotado no presente trabalho a partir do materialismo histórico dialético, a apresentação dos resultados e discussões, responde às categorias de análise a partir de uma perspectiva ampliada e contextualizada históricosocialmente, envolvendo todo o processo de desenvolvimento pedagógico que permeou a construção do caderno técnico e da formação presencial em questão

As categorias de análise que emergiram da entrevista com informante-chave incluíram: Bases Epistemológicas do Academia da Saúde, Experiências prévias nos Polos, Processo de elaboração do caderno, Atualização do caderno, etapas de construção da formação presencial e metodologias utilizadas. Tais achados foram complementados pelos dados coletados a partir da observação-participante e da análise documental realizadas pelas pesquisadoras.

Dessa forma, com base na descrição do processo de construção do caderno técnico e a partir do referencial teórico da investigação temática proposta por Freire (1987), foi possível estabelecer uma comparação entre as etapas implementadas, conforme descrito no esquema abaixo: 
Etapas de investigação temática propostas por Freire

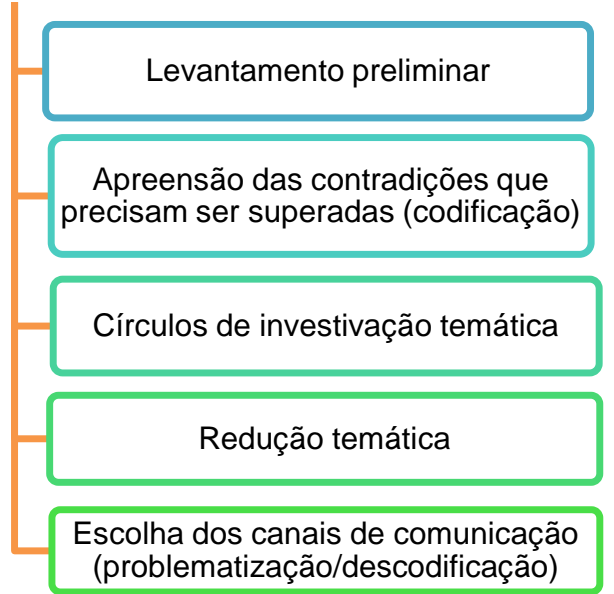

Etapas de construção do conteúdo programático do caderno técnico

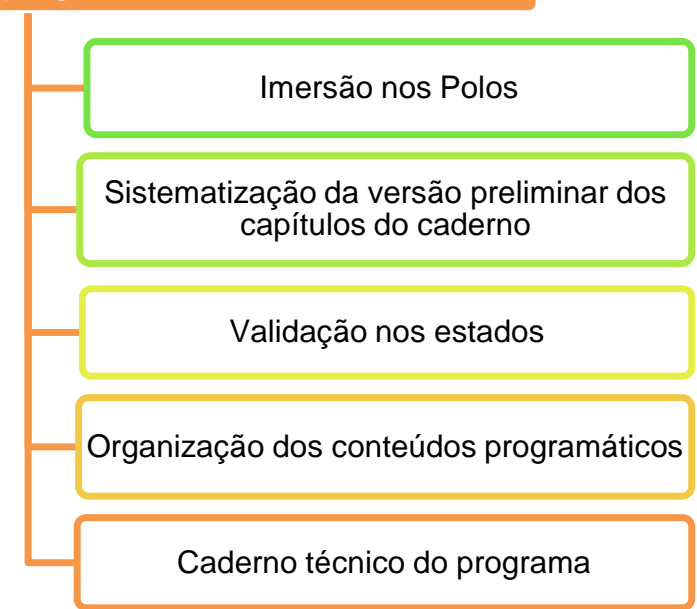

Fig. 1. Análise comparativa entre o método de investigação temática proposto por Freire e o processo de construção dos conteúdos programáticos do caderno técnico, baseado na entrevista com informante-chave. Fonte: REIS, G.A., 2020.

A partir dos registros das atas de reuniões do projeto e da observação-participante realizada pela pesquisadora, foi possível resgatar a etapas do planejamento e elaboração da formação presencial, onde verificou-se a realização de encontros formativos de planejamento entre a equipe responsável pelo projeto na UFT e o Ministério da Saúde para aproximação e aprofundamento com o universo do programa, a partir dos quais foi elaborada uma matriz pedagógica para a formação, fundamentada nos conteúdos programáticos do caderno técnico e traduzida em objetivos de aprendizagem definidos previamente à construção das oficinas.

Tanto a carga horária, como as metodologias e os tempos pedagógicos da formação foram definidos a partir destes encontros formativos da equipe e com base na matriz pedagógica elaborada previamente. Após sistematizada a versão preliminar da formação, foi realizada uma formação piloto com a finalidade de validar esse processo junto ao público-alvo (profissionais e gestores municipais). Esta etapa resultou na revisão e reformulação da formação que foi posteriormente aplicada nas oito microrregiões de saúde do estado do Tocantins.

Sendo assim, foi possível verificar uma aproximação do percurso metodológico empregado pelos pesquisadores docentes com o método de investigação temática proposto por Freire (1987), conforme comparativo descrito abaixo: 


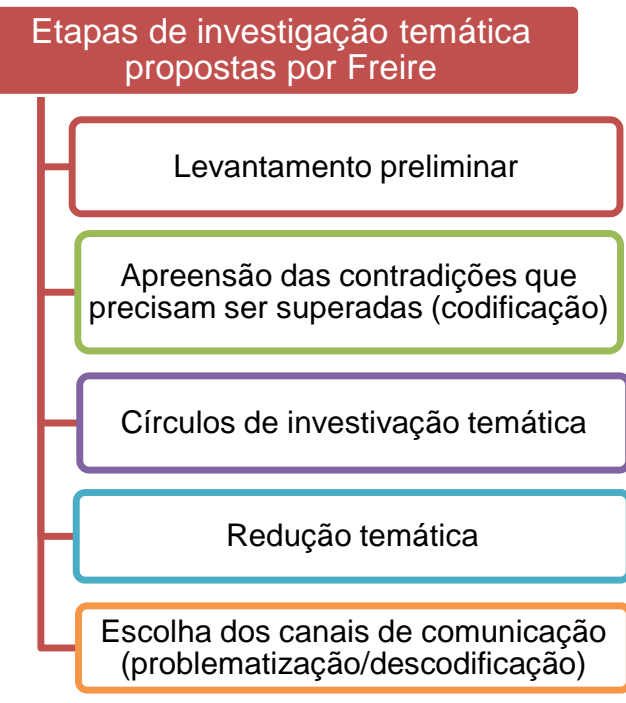

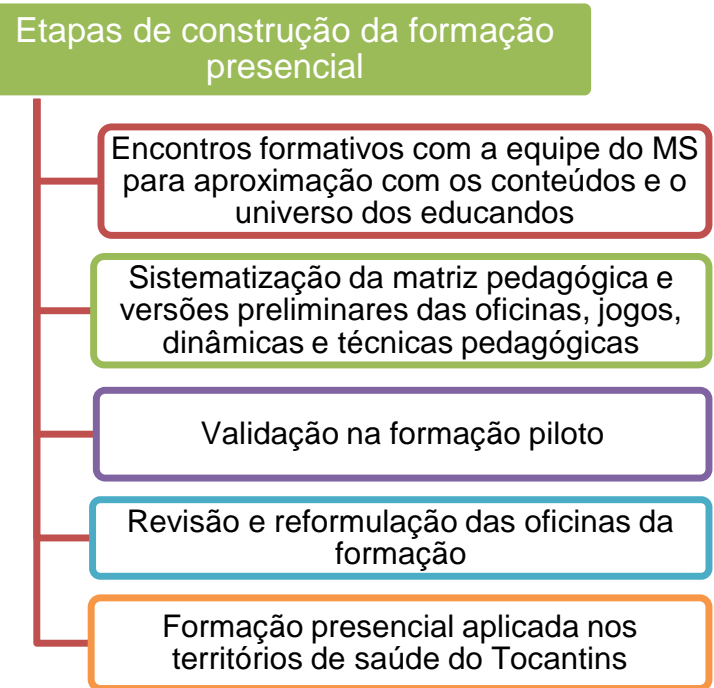

Etapas de construção da formação presencial

territórios de saúde do Tocantins

Fig. 2. Análise comparativa entre o método de investigação temática proposto por Freire e o percurso pedagógico de construção da formação presencial. Fonte: REIS, G.A., 2020.

Por fim, a observação-participante realizada pelas pesquisadoras aliada à análise documental dos relatórios de campo dos tutores permitiu investigar as metodologias e as abordagens pedagógicas empregadas na elaboração das oficinas da formação presencial. Sobre os recursos pedagógicos verificou-se a utilização de metodologias ativas de problematização da realidade, tendo como um dos pontos altos do processo pedagógico desenvolvido, a criação de jogos e a utilização de dinâmicas educacionais para trabalhar a aprendizagem dos conteúdos abordados no caderno técnico, além disso, outras estratégias pedagógicas de problematização da realidade também foram utilizadas, envolvendo atividades práticas e discussões coletivas realizadas no "círculo de cultura" com os participantes da formação.

\section{Conclusões}

A estratégia de triangulação de métodos qualitativos, permitiu investigar a elaboração do caderno técnico e da formação presencial voltados ao Programa Academia da Saúde. Neste sentido, a entrevista com informante-chave (IC) foi fundamental para a análise do processo de construção do caderno técnico do programa Academia da Saúde, por se tratar de um processo retrospectivo da pesquisa, permitindo assim inferir sobre a relação com a pedagogia freiriana e os pressupostos da educação permanente em saúde.

Cabe ressaltar que a informante-chave esteve à frente do Programa Academia da Saúde desde sua criação até a celebração da carta acordo firmada entre MS/UFT em 2019, tendo liderado o grupo responsável pela elaboração do Caderno Técnico de Apoio à Implantação e Implementação do Programa Academia da Saúde.

Em relação à construção do processo pedagógico que resultou na elaboração da formação presencial voltada ao programa Academia da Saúde e baseada nos conteúdos do caderno técnico, ressalta-se que a combinação dos métodos de observação-participante, aliada à análise documental das atas de reuniões e relatórios de campo dos tutores, permitiu que as pesquisadoras vivenciassem o processo pedagógico do início ao fim, sistematizando as etapas percorridas enquanto participavam das atividades juntamente com o restante da equipe de educadores.

Assim, foi possível demonstrar a aproximação por parte da equipe de educadores com o universo dos educandos, desde a sistematização da matriz pedagógica até a elaboração de oficinas baseadas em metodologias ativas de ensino-aprendizagem. 
Ademais, a validação junto ao público-alvo por meio da "formação piloto" agregou maior confiabilidade e pertinência ao processo formativo, demarcando um compromisso da equipe pedagógica com um processo educacional conectado aos anseios e necessidades dos educandos, estando assim alinhado aos referenciais de Freire e da educação permanente em saúde.

Podemos afirmar que a triangulação dos métodos utilizados para a avaliação deste projeto específico de educação permanente em saúde, possibilitou a análise, compreensão e avaliação do método em várias facetas, convergindo em uma intersecção entre os dados, culminando em aprendizado participativo das pessoas envolvidas.

A partir desta pesquisa foi possível vislumbrar as potencialidades da estratégia de triangulação de métodos de investigação qualitativa, como uma alternativa eficiente para abarcar grandes pesquisas, especialmente na avaliação de programas de educação permanente em saúde.

\section{Referências}

Bardin, L (2011). Análise de Conteúdo. São Paulo: Edições 70.

Bisol, C. A (2012). Estratégias de pesquisa em contextos de diversidade cultural: entrevistas de listagem livre, entrevistas com informantes-chave e grupos focais. Estudos de psicologia, Campinas, v. 29, supl. 1, p. 719-726.

Brasil (2011). Ministério da Saúde. Institui o Programa Academia da Saúde. Portaria no 719/GM/MS de 07 de abril de 2011. Brasília.

Brasil (2004). Ministério da Saúde. Política Nacional de Educação Permanente em Saúde. Portaria n 198/GM/MS de 13 de fevereiro de 2004. Brasília.

Brasil (2018). Ministério da Saúde. Programa Academia da Saúde: caderno técnico de apoio a implantação e implementação. Brasília: Ministério da Saúde, p. 220.

Brasil (2012). Ministério da Saúde. Resolução CNS no 466 de 12 de dezembro de 2012. Brasília.

Buss, P.M. \& Carvalho, A.I (2009). Desenvolvimento da promoção da saúde no Brasil nos últimos vinte anos (1988-2008). Ciênc. saúde coletiva, Rio de Janeiro, v. 14, n. 6, p. 2305-2316.

Freire, P (1987). Pedagogia do oprimido. 17ª edição, Rio de Janeiro: Paz e Terra.

Lüdke, M. \& André, M.E.D.A (1986). Pesquisa em educação: Abordagens Qualitativas. São Paulo: EPU.

Minayo M.C.S., Assis S.G. \& Souza E.R. (2005). Avaliação por triangulação de métodos: abordagem de programas sociais. Rio de Janeiro: editora Fiocruz, p. 244.

Reis, G.A. (2020). Programa Academia da Saúde: Análise do processo pedagógico de educação permanente desenvolvido na formação presencial aplicada no estado do Tocantins. 2020. 121 f. Dissertação (Mestrado em Ciências da Saúde) - Universidade Federal do Tocantins, Palmas.

Souza, J., Kantorski, L.P. \& Luis, M.A.V. (2011). Análise documental e observação participante na pesquisa em saúde mental. Revista Baiana de Enfermagem, Salvador, v. 25, n. 2, p. 221228, maio-ago. 